\title{
Retenue dans les moeurs et maîtrise de la violence politique. La thèse de Norbert Elias
}

\section{Claudine Haroche}

\section{(2) OpenEdition \\ Journals}

Édition électronique

URL : http://journals.openedition.org/conflits/239

DOI : $10.4000 /$ conflits.239

ISSN : $1777-5345$

Éditeur :

CCLS - Centre d'études sur les conflits lilberté et sécurité, L'Harmattan

Édition imprimée

Date de publication : 15 mai 1993

ISSN : 1157-996X

Référence électronique

Claudine Haroche, «Retenue dans les moeurs et maîtrise de la violence politique. La thèse de Norbert Elias », Cultures \& Conflits [En ligne], 09-10 | printemps-été 1993, mis en ligne le 04 mars 2005, consulté le 30 mars 2021. URL : http://journals.openedition.org/conflits/239 ; DOI : https://doi.org/10.4000/ conflits.239

Ce document a été généré automatiquement le 30 mars 2021

Creative Commons License 


\title{
Retenue dans les moeurs et maîtrise de la violence politique. La thèse de Norbert Elias
}

\author{
Claudine Haroche
}

1 Les thèses de Norbert Elias en matière de violence sont connues. Aussi se bornera-t-on ici à les rappeler brièvement. Elias fait du niveau de maîtrise de l'affectivité dans une société un élément déterminant du procès de civilisation. C'est en effet dans la mise à l'écart de l'agressivité et de la violence par le contrôle social "ancré dans l'organisation étatique" qu'il voit la marque d'une "société civilisée". Elias décrit d'abord le climat psychologique de la société médiévale où l'homme doit pouvoir et vouloir infliger la mort ou encore accepter de risquer sa vie jusqu'à la perdre. "La seule menace, le seul danger qui pesaient sur ces hommes étaient ceux d'être terrassés par un adversaire plus fort qu'eux"1. La vie du guerrier du Moyen Age est un combat permanent. Les manifestations de cruauté physique, le plaisir que les hommes pouvaient y prendre, n'étaient pas jugées sévèrement: elles étaient même tenues pour parfaitement légitimes. L'homme médiéval incapable d'aimer et de haïr avec une même passion, "était aussi perdu que le sera dans la société de cour l'homme incapable de réfréner ses passions, de dissimuler ses réactions affectives, de se comporter en homme civilisé". Elias offre alors l'explication suivante : "Dans un cas comme dans l'autre, c'est toujours la structure de la société qui postule et cultive une certaine forme de répression affective" ${ }^{2}$. Elias entreprend d'élucider l'origine des structures sociales responsables de nouvelles structures émotionnelles. La raison pour laquelle l'homme médiéval va se transformer en homme de cour s'explique par le mécanisme de la monopolisation par l'Etat de la violence. Elias explique longuement les modalités et les raisons qui ont présidé à la formation de ces monopoles depuis leur apparition en France au XIème siècle ${ }^{3}$. Le pourquoi d'abord: à l'origine de la détention par le pouvoir central du monopole de la violence: "un accroissement de la population (qui) entraîne une augmentation des besoins en terres..." ${ }^{4}$. Le comment ensuite: "Le processus de la formation de monopoles obéit, dans ses grandes lignes, à des lois structurales extrêmement précises...toute compétition sociale libre et éliminatoire tend à la 
formation de monopoles"5. Mais la libre compétition va reculer progressivement devant une compétition régie par certaines règles. Elias illustre alors son propos en comparant la situation de la noblesse féodale libre à celle de la noblesse de cour, évolution qu'il décrit comme un mouvement général vers la civilisation. Pour la première, "l'emploi de la violence physique est un moyen de combat indispensable à la conquête...." ${ }^{6}$. Par contre dans la noblesse de cour le recours à la violence pure et simple est à peu près exclu... Les moyens de la compétition se sont raffinés et sublimés ; la dépendance des individus par rapport au détenteur du monopole impose à chacun une plus grande retenue dans ses manifestations émotionnelles; les individus sont tiraillés entre la résistance aux contraintes auxquelles ils se trouvent exposés, l'horreur que leur inspire leur dépendance et leur état de soumission, la nostalgie de la compétition libre d'une part, et la fierté de la maitrise de soi qu'ils ont réussi à s'imposer, les plaisirs nouveaux qu'elle leur propose, de l'autre. En d'autres termes, nous avons affaire à un mouvement général vers la civilisation?7. "L'Etat européen classique" écrit ainsi Carl Schmitt dans une analyse remarquablement pénétrante "avait réussi cette chose tout à fait invraisemblable qui fut d'instaurer la paix à l'intérieur et d'exclure l'hostilité en tant que concept du droit. Il avait réussi à supprimer la guerre privée, institution du droit médiéval, à mettre fin aux guerres de religion du XVI ème et du XVII ème siècle, que les deux camps considéraient comme des guerres particulièrement justes, et à établir la tranquillité, la sécurité et l'ordre dans les limites de son territoire. On sait que cette formule "la tranquillité, la sécurité et l'ordre" servait à définir la police. Et en effet au sein de cet Etat, il n'y avait plus qu'une police, la politique en était absente; à moins que l'on ne désigne par ce terme les intrigues de cour, les rivalités, les frondes et les tentatives de rébellion des malcontents, bref, les 'troubles'" 8 . Un peu plus loin Schmitt ajoute encore " Ces bornes où l'on renferme la guerre et sa nette délimitation impliquent que l'hostilité n'a plus qu'une portée relative. Toute relativisation de cette sorte est un grand progrès vers plus d'humanité" 9. Elias voit ainsi les origines de ce changement d'économie pulsionnelle dans le fait que désormais "le monopole de la contrainte physique est assuré par le pouvoir central, l'individu n'a plus le droit de se livrer au plaisir de l'attaque directe" ${ }^{110}$. Le corps à corps par lequel s'exprimait la violence physique dans la société médiévale jusqu'à la Renaissance s'efface maintenant devant le face à face : le processus de monopolisation de la force au profit de l'Etat s'accompagne d'une intériorisation des contraintes, d'une auto-contrainte et d'une maîtrise de soi qui contribuent à la pacification des espaces sociaux. Ailleurs Elias nuance quelque peu cette opposition radicale qu'il établissait entre l'homme du Moyen Age et l'homme de cour, entre le chevalier libre et le chevalier de cour rapidement transformé en courtisan. Il remarque en effet que la maîtrise de soi ${ }^{11}$ existait déjà "dans la société de guerriers médiévale et dans d'autres sociétés dépourvues d'administrations monopolistiques de la force militaire et policière. Le mécanisme psychique de l'autocontrôle, le "surmoi", la "conscience"...est inculqué, imposé et entretenu dans une société de guerriers dans la perspective de l'exercice de la violence physique; les structures d'un tel autocontrôle répondent aux exigences d'une vie faite de contrastes et de brusques sautes d'humeur. Quand on les compare aux mécanismes d'autocontrainte des sociétés pacifiées, on constate qu'elles sont moins rigoureuses, moins stables et qu'elles laissent plus de place à de brusques décharges émotionnelles". Une plus grande retenue se fait jour dans les liens sociaux, qui produit des effets dans les moeurs. Evoquant la vie nouvelle des chevaliers à la cour, Elias souligne ainsi que "le 
nouvel espace vital, la nouvelle forme d'intégration imposent aux hommes une autodiscipline nouvelle, une retenue infiniment plus forte" ${ }^{12}$. Laisser parler son corps, exprimer trop ouvertement ses émotions et ses sentiments en société sont ainsi des attitudes à proscrire. Le "voir" est alors privilégié au détriment du contact physique. Les préceptes en matière d'éducation qui entendent inciter à ne plus toucher mais à se contenter de regarder, "visent à transformer les plaisirs d'une agressivité active en plaisir passif, codifié, en simple plaisir des yeux"13. Il faut désormais, comme le dit en 1774 dans sa Civilité chrétienne J.B. de La Salle, "apprendre (aux enfants) à ne toucher que des yeux tout ce qu'ils voient"14. Une distance s'instaure ainsi entre les individus; les règles de la civilité imposent une plus grande réserve physique. En 1939, lorsqu'il écrit La civilisation des moeurs, Elias posait donc clairement que la retenue écarte la violence physique ; mais aussi que cette même retenue peut parfaitement accompagner d'autres formes de violence, psychologique et symbolique, dans lesquelles l'habileté, la prudence, la réticence, le calcul, la manipulation, la dissimulation sont essentiels. S'il est vrai que "la violence physique a été bannie des relations humaines, que même le duel a été interdit par la loi, l'homme n'en exerce pas moins sur ses semblables toutes sortes de contraintes et de violences". Et modulant quelque peu ce qu'il dit souvent ailleurs lorsqu'il parle d'espaces pacifiés, "la vie dans cette sphère n'est pas une vie paisible"15. Ainsi Elias souligne-t-il que l'étiquette et le cérémonial de la société de cour absolutiste ont une fonction sociale et politique non dénuée de violence: dans l'étiquette "chaque détail a la valeur d'une arme dans la lutte pour le prestige" ${ }^{16}$. D'autres formes de violence infiniment plus feutrées, compatibles avec la modération, la réserve, la maîtrise de soi se font jour dans ces espaces pacifiés que sont les cours princières, puis, plus nettement encore, dans la société de cour au XVIIème siècle. Ainsi Rousseau soulignera-t-il un peu plus tard ce glissement en notant avec subtilité : "On n'outragera point grossièrement son ennemi, mais on le calomniera avec adresse" ${ }^{17}$. Un ensemble de questions se pose dès lors: les manières, la retenue peuvent-elles réellement entraver la violence, l'écarter, la supprimer? Ne font-elles pas au mieux que la dissimuler? Plus encore : bien loin de simplement s'accompagner de violence, la retenue ne peut-elle pas parfois naître de la violence, ou même la susciter ? Pour tenter de répondre à ces questions arrêtons-nous à nouveau sur certains des écrits d'Elias. Au milieu des années 1980, dans une série d'entretiens autobiographiques, il livre en effet un ensemble de remarques particulièrement éclairantes sur la question de la violence politique - remarques tout à la fois passionnantes et surprenantes, parfois même un peu déconcertantes, qu'il inscrit dans la longue durée ${ }^{18}$. Quelques années auparavant il avait déjà abordé la question du lien entre violence et culture politique en Allemagne, à un autre niveau, en offrant une explication plus contemporaine, relevant du sociologique, du politique et de la morale ${ }^{19}$. Il expliquait l'indifférence morale des élites bourgeoises en particulier, l'acceptation ou du moins la tolérance d'une certaine violence politique illégale, la défaillance de la morale traditionnelle pendant la République de Weimar, par le progrès des institutions privées, des milices de voisinage et la "désintégration du monopole de l'Etat sur la violence" . Une indifférence morale qui présuppose, comme le dit très justement Bernd Weisbrod ${ }^{20}$, une "désintégration de la conscience". C'est sur ces entretiens biographiques que nous nous arrêterons maintenant. Le sociologue y rappelle comment, ayant fui l'Allemagne au milieu des années 1930, il s'est installé à Londres. En s'interrogeant sur les usages, les traditions, les coutumes, sur ce que l'on désigne en particulier par culture politique, il confie qu'il aura plus tard le sentiment qu'il cherchait, à la veille de la seconde guerre mondiale, à 
élucider le problème de la brutalité et de la violence, physique et symbolique, qui ne cessaient alors de progresser en Allemagne, par l'ébauche d'une histoire comparée des normes et des règles qui sous-tendaient les comportements des hommes dans les sociétés européennes d'autrefois. Un jour, rappelle-t-il, il "tombe" sur une piste qui lui paraît prometteuse: les traités d'étiquette. Il entreprend alors de retracer dans la perspective d'une psychologie historique des comportements collectifs, à partir des manuels de civilitéé ${ }^{21}$ l'histoire de ces règles et de ces rituels qui ont pour objet le gouvernement des corps et des conduites. Puisque La Civilisation des moeurs paraît en 1939, près de cinquante ans la séparent donc de ces Entretiens et Notes biographiques. Le propos a quelque peu changé. C'est en effet là, pour la première fois semble-t-il, qu'Elias reconnait un lien aussi explicite entre la situation politique en Allemagne, la montée de la violence et son propre intérêt pour les traités de civilité. En outre, là où à la fin des années 1930, il posait que la retenue, la maîtrise de soi relèvent des liens entre économie pulsionnelle, structures émotionnelles et structures sociales, il aperçoit désormais beaucoup plus clairement les effets politiques d'une telle retenue ou plutôt de son absence, qui entraîneraient violence sociale et politique. A la fin des années 1980, il voit ainsi dans le choix initial de son objet de réflexion l'histoire des moeurs et des manières, l'histoire des règles de civilité, des normes de comportement, de la retenue en particulier, des raisons politiques. De la maîtrise de soi il fait donc maintenant une question fondamentalement politique ${ }^{22}$. Revenant sur des objets auxquels il avait consacré les deux premiers chapitres de La civilisation des moeurs, l'importance de la civilisation en France et en Angleterre, qu'il oppose au rôle de la culture en Allemagne, Elias remarque alors que les manières allemandes et françaises sont différentes, beaucoup moins civilisées selon lui, en Allemagne qu'en France. Il va même jusqu'à insister sur leur grossièreté. Le comportement germanique se caractériserait à ses yeux, par un manque de maîtrise de soi, parfois extrême. Il va alors l'expliquer, en particulier, par le fait que "la barrière qui séparait la noblesse de la bourgeoisie était beaucoup plus élevée...qu'elle ne l'était en France". Un peu plus loin, il ajoute "qu'une fusion s'est produite en France et en Angleterre entre la morale bourgeoise et les bonnes manières aristocratiques" tandis que "le caractère national allemand a été marqué beaucoup plus profondément par les classes moyennes" ${ }^{23}$. Pour lui l'importance des manières dans l'histoire nationale respective de l'Allemagne, de la France et de l'Angleterre, semble donc cruciale. Elias va pourtant aller au delà de ce projet de psychologie historique auquel il voulait travailler au début des années 1930. Pour tenter d'expliquer la violence politique il fait appel à une hypothèse psychanalytique. Après la défaite de 1918, il remarque: "On se retrouva soudain avec une structure de personnalité fondée sur le principe de l'obéissance et de la discipline à l'égard d'un monarque puissant, dans un Etat où comme il le dit lui-même - j'exagère un peu - la partie externe du surmoi avait disparu". Evoquant la République de Weimar, Elias souligne que "de nombreux Allemands commencèrent à appeler de leurs voeux un homme fort qui leur redonnerait la possibilité de se dominer. Ils n'avaient pas appris à faire cela par eux mêmes. Un tel régime contribue à développer un surmoi dans certaines sphères seulement. Il faut donc compléter la notion freudienne de surmoi en indiquant que le surmoi peut être irrégulier ou fragmentaire. Il peut par exemple être très présent au niveau de la famille...et être absent en même temps dans le domaine politique. Et c'est ce qui se passait effectivement en Allemagne" ${ }^{24}$. En suggérant que le système politique sous lequel les uns et les autres ont vécu aurait encouragé des traits de personnalité, des tendances profondément différentes dans les économies 
psychiques ${ }^{25}$, Elias va avec le temps entrevoir dans la retenue bien plus qu'une question psychologique et sociologique touchant simplement aux moeurs, bien davantage qu'une question strictement anthropologique: une question fondamentalement anthropologique et politique. Il semble ainsi qu'en 1980 Elias réintroduise le contexte politique, prenant en compte la brutalité, la violence à l'intérieur même de son étude sur la civilité, les manières, la civilisation des moeurs et par les moeurs. Ou encore qu'il réinscrive dans la perspective d'une anthropologie politique l'hostilité, l'agressivité, la cruauté, les instincts mal dominés. On peut alors se demander si, en 1939, lorsqu'il écrit La civilisation des moeurs, Elias n'a pas oublié la dimension fondatrice de l'hostilité, de la violence dans les relations sociales pacifiées par le monopole étatique de la violence, prenant alors au pied de la lettre cette approche qui a tendance à confondre comportement policé et politique ${ }^{26}$. En analysant comme il le fait les rapports entre l'aristocratie et la bourgeoisie en Allemagne, et plus largement en Europe, les conséquences de ces rapports sur les classes moyennes et le prolétariat, Elias rappelle l'intérêt qu'il peut y avoir à prendre en compte les manières, les usages et les comportements psychologiques pour éclairer certains aspects des cultures nationales. Dans le fait que les classes moyennes allemandes n'ont jamais adopté les modèles aristocratiques, il décèlera en conséquence l'un des facteurs susceptibles d'expliquer la violence des comportements allemands. Il est alors intéressant de mettre en rapport les thèses d'Elias, qui s'inscrivent dans la longue durée, et celles de Weisbrod qui étudie le rapport entre violence et culture politique en Allemagne entre les deux guerres. Celuici pose que le processus qui devait conduire à vider les normes sociales bourgeoises de leur substance avait commencé bien avant la terreur engendrée par le régime nazi. Ce processus, dit Weisbrod, "s'explique bien plus par une culture politique propre qui avait intégré depuis la guerre, une acceptation de la violence politique......qu'on trouve également en germes dans la mentalité d'auto-défense des corps francs et des milices de voisinage" précisant en effet que " sous le couvert de l'autodéfense civile, les milices de voisinage constituèrent un moyen important d'initiation à des formes violentes de mobilisation politique......qui permirent ensuite la banalisation de la violence dans la culture politique de Weimar"27.

\section{LA FORCE DES RITUELS ET DES FORMES}

Elias voit dans les règles de la civilité et de la politesse le concours de chaque individu au maintien de l'ordre dans une société, et l'expression de la nécessité des formes dans la vie sociale. Goffman examinant lui aussi la question de la tenue et de la déférence dit en substance la même chose qu'Elias dans le cadre d'une psychologie interactionniste ou encore, comme il le dit lui-même, d'une "version modernisée de la psychologie sociale de Durkheim". De la tenue et de la déférence Goffman fait des éléments de tout "moi" en société, ce qu'il désigne comme le "moi cérémoniel"; tout comportement social et public est perçu par lui comme un comportement cérémoniel, ce qui le conduit à définir une société comme "un système d'accords de non empiétements" ${ }^{28}$. Il faut alors s'arrêter sur la vertu spécifique des formes: structurer et mettre à distance ; organiser et imposer. Expliciter des rituels, reconnus et acceptés, permet de réguler les comportements, gouverner les conduites, prévenir le désordre et la violence, latents en toute société, en imposant une distance entre individus qui écarte la violence physique 
du corps à corps. Mettre des formes, poser des limites, instaurer des frontières entre chaque individu, est un moyen de pacifier les rapports sociaux. Pourtant la force de la forme implique l'existence d'une violence potentielle au niveau des effets mais, tout aussi bien, des fondements. Les formes et le respect des formes dans une société (qui constituent des moyens de pacification à l'échelle collective, nationale ou internationale) tendent à écarter la violence physique mais accompagnent, confortent parfois, des expressions de violence symbolique. "Plus la situation sera grosse de violence à l'état potentiel", note fort justement P. Bourdieu, "plus il faudra mettre des formes, plus la conduite librement confiée aux improvisations de l'habitus cédera la place à la conduite expressément réglée par un rituel méthodiquement institué, voire codifié. Il suffit de penser au langage diplomatique ou aux règles protocolaires qui régissent les préséances et les bienséances dans les situations officielles"29. "Codifier", ajoute-t-il encore, "c'est à la fois mettre en forme et mettre des formes". Il y a, conclutil, "une vertu propre de la forme" ${ }^{30}$. Ainsi les formes, qu'il faille entendre sous ce terme les rituels au sens large, les cérémonies, le protocole, les règles de la civilité, les normes de comportements quotidiens en société, les lois civiles, juridiques ou politiques, sont toutes fondamentalement des moyens d'instaurer et de maintenir des distances, de lutter contre la violence physique, de tenter de la contrôler, de la réglementer, de la réduire voire de la supprimer par la réprobation morale ou les sanctions juridiques. Il convient sans doute de distinguer le droit des simples règles de la politesse qui régissent en particulier les relations personnelles et psychologiques. Leurs contraintes ne sont pas de l'ordre de la répression stricto sensu : aucune sanction autre que morale ne vient punir l'absence de politesse. Seules, injures et insultes font l'objet d'une législation. "En punissant l'injure, la diffamation, les propos racistes, les violences et les voies de fait entre autres, le Code Pénal sanctionne des comportements qui sont de l'ordre de l'impolitesse, c'est à dire du non respect de l'autre dans son être physique et moral" ${ }^{11}$. Il faut ainsi voir dans les formes différentes composantes : ce qui structure et met de l'ordre dans une société, ce qui impose des égards dans les rapports entre individus; mais aussi ce qui est tenu pour superficiel, voire faux et mensonger. Ceci peut expliquer la violence des attaques contre la politesse de cour sous l'Ancien régime auxquels se livrent les moralistes avec la Bruyère en particulier, les politiques avec Rousseau. En 1750 dans le Discours sur les Sciences et les Arts, Rousseau la dénonce en ces termes : "Sans cesse la politesse exige, la bienséance ordonne : sans cesse on suit des usages, jamais son propre génie. On n'ose plus paraître ce qu'on est; et dans cette contrainte perpétuelle....on ne saura donc jamais bien à qui l'on a affaire.....Quel cortège de vices n'accompagnera point cette incertitude ? Plus d'amitiés sincères ; plus d'estime réelle; plus de confiance fondée. Les soupçons, les ombrages, les craintes, la froideur, la réserve, la haine, la trahison se cacheront sans cesse sous ce voile uniforme et perfide de politesse, sous cette urbanité si vantée que nous devons aux lumières de notre siècle" ${ }^{12}$.

\section{SE DOMINER, DOMINER AUTRUI}

Dans les règles de la civilité et de la politesse, Elias veut voir la possibilité et le moyen de réaffirmer une éthique du comportement de soi à l'endroit d'autrui. Il ne s'est guère arrêté sur le fait que les règles de la civilité sont des formes qui non seulement 
entravent la violence mais parfois l'accompagnent. Elias ne s'est donc pas montré très sensible à la dimension paradoxale de la retenue et de la contenance. Or c'est, nous semble-t-il, un des aspects par où prolonger et approfondir la composante anthropologique et politique de la retenue : il faut en effet $\mathrm{y}$ voir tout à la fois le fait de se dominer, de respecter les autres, mais encore de dominer autrui. Pouvoir exercé sur soi comme condition nécessaire au respect d'autrui ; mais dans le même temps pouvoir exercé sur soi comme élément indispensable au gouvernement des autres. Et il faut ici se remémorer cette formule de Louis XIV dans ses Mémoires : "Celui qui ne produit rien de soi fait paraître beaucoup davantage ce qu'il exécute". Il faut savoir se gouverner pour gouverner les autres avec mesure; il faut encore savoir se gouverner pour dominer les autres, leur imposer un pouvoir par la douceur, la raison ou la violence. Les traités de civilité, érasmienne, chrétienne ou baroque auxquels s'est intéressé Elias, présentent alors une proximité surprenante avec les "miroirs des princes" des XVIe et XVIIe siècle, ces traités destinés à l'éducation des souverains. Un ensemble d'écrits pédagogiques que La Mothe le Vayer dédie au Dauphin ${ }^{33}$ rappelle ainsi : "Il est du tout nécessaire qu'un homme scache se gouverner soy mesme devant que de commander aux autres, soit comme père de famille, ce qui est de l'Oeconomie, soit comme souverain, magistrat ou ministre d'Estat, ce qui regarde la politique" ${ }^{34}$. Savoir conduire une famille et bien gouverner un peuple relèvent fondamentalement d'une même exigence, d'un même principe, d'une même qualité : se dominer, savoir se gouverner soi même. "Qu'on ne présume pas", écrit encore La Mothe le Vayer, "qu'une personne incapable de bien ordonner son domestique, doive réussir dans le gouvernement public" $^{135}$. Qu'il s'agisse donc d'économie domestique ou politique, le gouvernement de soi est indispensable au gouvernement des autres. Et la plupart des '"miroirs des princes" ne cessent de le répéter : le gouvernement du prince par lui-même est la condition du gouvernement des sujets. A la fin du XVIIème siècle Fénelon faisait paraître ses Directions pour la conscience d'un Roi. Il y témoignait d'une préoccupation identique : l'empire sur soi est indissociable de l'empire sur les autres. Le Dauphin est encore enfant, et Fénelon s'adresse à lui en ces termes:"Un des plus grands malheurs qui vous pût arriver, serait d'être maître des autres, dans un âge où vous l'êtes encore si peu de vous même" ${ }^{136}$. Traités d'éducation des princes ou manuels de civilité, une même exigence se laisse donc entrevoir : il importe d'apprendre à se dominer, à contenir ses passions pour maintenir l'ordre chrétien, social et politique. Cette dimension paradoxale et ambiguë de la retenue, de la maîtrise de soi nous semble offrir un prolongement et un approfondissement des travaux d'Elias dans une perspective d'anthropologie politique. Il faut alors s'arrêter sur Carl Schmitt qui décèle dans toute théorie politique des fondements anthropologiques. Lisant attentivement l'ouvrage de Spranger : Lebensform (Formes de vie), en particulier le chapitre "Der Machtmensch" (le type dominateur), Schmitt y remarque que ce chapitre présente toute la réalité existentielle du politique. Ainsi relève-t-il cette phrase extraite de Lebensform: "La dignité du type dominateur semble croître en même temps que sa sphère d'influence". Schmitt remarque qu'elle concerne "un phénomène qui se situe dans la sphère du politique et qui, de ce fait, ne souffre d'autre interprétation que politique, en ce sens qu'elle est une application de la thèse selon laquelle le point où un phénomène devient politique est déterminé par l'intensité de la distanciation qui commande les associations et les dissociations décisives" ${ }^{37}$. Ailleurs Schmitt note que Plessner est le premier philosophe moderne à avoir tenté d'élaborer une anthropologie politique de grande envergure, et remarque alors quelque chose qui rappelle tant l'instauration 
d'une distance entre individus chez Elias que le moi et le comportement cérémoniel chez Goffman. Se référant à Plessner, "l'homme, écrit ainsi Schmitt, est primordialement un être de distanciation dont la nature demeure indéfinie et insondable" ${ }^{138}$.

\section{LA FORME COMME FORCE ET COMME ESTHETIQUE}

Bien des écrits contemporains dénoncent l'absence de politesse et de civilité comme étant la marque de sociétés démocratiques qui, se voulant égalitaires, croient devoir refuser toute formes et formalités superflues, toute entrave à ces liens authentiques dont chaque individu aurait besoin. De telles sociétés finissent par imposer à l'individu une violence qui prend la forme d'injonctions à l'authenticité et à la transparence. L'absence de politesse et de formes conduirait ainsi à l'incivilité et à la violence ${ }^{39}$. Il est dès lors intéressant de se tourner vers des sociétés où la politesse et l'étiquette jouent un rôle crucial. M. Miho pour la société japonaise, J. Dulck pour la société anglaise s'interrogent ainsi sur le point de savoir s'il existe une corrélation entre intensité de la violence sociale et importance des codes de savoir vivre ${ }^{40}$. Tous deux posent que la politesse ne s'opposerait pas à la violence; elle en serait, disent-ils, "la forme socialisée". Le Japon et l'Angleterre, sociétés policées et violentes, en témoigneraient ${ }^{41}$. Il y aurait ainsi, selon ces auteurs, une parenté profonde entre la politesse et la violence : la politesse naîtrait de la violence, du besoin de l'entraver ; et inversement, la violence naîtrait d'une politesse démesurée. L'excès de maîtrise de soi nécessité par l'excès de politesse constituerait une violence faite à soi-même et à autrui : il apparaîtrait non plus tant comme du respect à autrui que comme le produit d'une violence refoulée. Au delà du souci des contenances extérieures, des idéaux de justice, de l'expression de codes et des normes, c'est le fait d'apercevoir dans la politesse comme dans la violence une dimension esthétique, de la beauté, de l'élégance, qui semble fondamental à $\mathrm{Miho}^{42}$. En faisant de la politesse "un geste concret que l'on appréhende comme beau en fonction de règles codifiées" ${ }^{43}$, la forme et les apparences deviennent en effet essentielles devant un sens authentique et caché : l'être intérieur. Les Japonais, s'interroge alors Miho, seraient-ils donc plus sensibles "aux manifestations concrètes, formelles et esthétiques de la politesse qu'à ses aspects moraux et éthiques" ?44. Quand "l'acte juste est celui qui est beau"45 remarque Miho, l'opposition entre la politesse et la violence tend à s'effacer: là où la politesse contribuait à l'ordre, l'harmonie, la paix, quand la beauté se substitue d'une certaine façon à la justice et à l'éthique, politesse et violence loin d'être disjointes peuvent alors, dominées par le souci esthétique, se voir confondues. Nous avons voulu retrouver chez Elias cette tradition qui, en particulier depuis Montesquieu et Tocqueville, entend éclairer le politique en s'interrogeant aussi sur l'histoire des manières et des comportements civils, pour en retrouver les fondements anthropologiques. Les origines historiques de ces préceptes de "retenue" permettent de repérer des rapports entre dispositions psychologiques, liens sociaux et comportements politiques. Nous avons voulu souligner les paradoxes de la retenue, nécessaire au respect d'autrui mais également présente dans sa domination. Et si les cérémonies, les rituels garantissent égards et respect à l'endroit d'autrui, ils permettent aussi de dissimuler l'absence de considération et de déférence : "Le comportement de déférence.....traduit peu les vrais 
sentiments de son auteur...Une telle facilité à manifester une déférence que l'on n'éprouve pas peut donner un sentiment d'autonomie interne qui permet de prendre ses distances par rapport à l'ordre cérémoniel, alors même qu'on le soutient en actes" ${ }^{46}$. Les formes imposent ainsi la mise à l'écart de la violence physique mais elles peuvent fort bien s'accompagner d'une violence symbolique et psychologique accrues.

\section{NOTES}

1. Elias (N.), La civilisation des moeurs, Paris, Calmann-Lévy, 1973, p.326.

2. Ibid. pp. 336-337.

3. Voir La dynamique de l'Occident, Paris, Calmann Lévy, 1975.

4. Ibid. pp.30-31.

5. Ibid. pp. 39-40.

6. Ibid. p.40.

7. Ibid. pp. 40-41.

8. La notion de politique, (1932), Trad.,.Flammarion, 1992, p.43.

9. op. cit.

10. Elias (N.), op. cit., p.200.

11. "Dans un certain sens", remarque Elias, "le champ de bataille a été transposé dans le for intérieur de l'homme. C'est là qu'il doit se colleter avec une partie des tensions et passions qui s'extériorisaient naguère dans les corps à corps où les hommes s'affrontaient directement" . La Dynamique de l'Occident, op. cit.p. 203.

12. La civilisation des moeurs, op. cit., p.363.

13. Ibid, p.339.

14. Les règles de la bienséance et de la civilité chrétienne. in J.P. Séguin. La bienséance, la civilité et la politesse enseignées aux enfants. Textes choisis. Ed. J.M. Place, 1992, p.69 et ss.

15. La dynamique de l'Occident, p. 242.

16. La société de cour, Paris, Calmann Levy, 1969, p : 108.

17. Rousseau, Discours sur les sciences et les arts. Paris, Garnier Flammarion, 1971,p.40. 18. Nous nous référons ici à la traduction française de l'ouvrage allemand Norbert Elias über sich selbst ( 1990), Norbert Elias par lui-même, Paris, Fayard, 1991. La première version paraît en Hollande sous le titre De Geschiedenis van Norbert Elias, Amsterdam, Meulenhoff, 1987.

19. N.Elias, "Zivilisation und Gewalt Über das Staatsmonopol der Körperlichen Gewalt und seine Durchbrechungen" in Studien über die Deurschen. Machtkämpfe und Habitusentwicklung im 19 und 20 Jahrhundert, Francfort, 1989.

20. Bernd Weisbrod, "Violence et culture politique en Allemagne entre les deux guerres" in XX ème siècle, Juin 1992.

21. Voir le long développement que Roger Chartier consacre à la notion de civilité dans son chapitre "Distinction et divulgation : la civilité et ses livres" in Lectures et Lecteurs dans la France d'Ancien Régime, Paris, Seuil,1987. 
22. De ce point de vue les travaux de L. Dumont, en particulier dans son dernier ouvrage où il aborde notamment les questions de l'éducation de soi, la formation de soi, la bildung dans la culture allemande [Homo Aequalis II, L'idéologie Allemande (FranceAllemagne et retour), Paris, Gallimard, 1991], nous semblent remarquablement éclairants pour comprendre une spécificité de la culture allemande et permettre ainsi de prolonger et d'approfondir les thèses d'Elias à partir d'une autre approche.

23. Norbert Elias par lui-même, op. cit., p 77.

24. Norbert Elias par lui-même, pp.77-78. Sur ces questions on renverra à E. Fromm, Escape from Freedom, Londres, Routledge and Kegan, 1942, et à Freud, Malaise dans la civilisation, 1929, Paris, P.U.F., 1971.

25. Idem. p. 75.

26. Voir Schmitt, La notion de politique, op. cit.

27. Bernd Weisbrod, op. cit., pp.113-125.

28. E. Goffman, Les rites d'interaction, Paris, Ed. Minuit, 1974, p.56.

29. P. Bourdieu, "Habitus, code, codification" in Actes de la recherche en sciences sociales, septembre $1986, \mathrm{n}^{\circ} 64, \mathrm{p} .41$.

30. Idem.

31. R. Dhoquois, "Sous contrat" in La Politesse, Ed. Autrement, Série Morales $n^{\circ} 2,1991$, p.112.

32. Rousseau, Discours sur les sciences et les arts, p.40.

33. La Politique du Prince, l'Oeconomique du Prince, 1653.

34. L'Oeconomique du prince, pp.1-2.

35. Idem, p. 3.

36. François de Salignac de la Mothe-Fénelon, Directions pour la conscience d'un Roi (composé pour l'instruction de Louis de France, duc de Bourgogne), pp. 1-2.

37. Schmitt, La notion de politique, op. cit., p.103.

38. Macht and menschliche Natur, 1931, p.103.

39. R. Sennett, The Fall of Public Man, New York, Vintage Books, 1975, (trad. française Les tyrannies de l'intimité, Paris, Seuil, 1979, p. 205).

40. M. Miho, J. Dulck, "Barrage contre la violence" in La politesse, op.cit.

41. Pourtant la corrélation est loin d'être toujours évidente. Les Etats Unis sont réputés pour leur violence, et non pour une politesse excessive. Il règne en effet dans les rapports sociaux une absence notoire de formalités. Sur ce point, voir en particulier C. Vouters, "Informalization and the civilizing process" dans P.R. Gleichman et al. ed., Human Figurations : essays for Norbert Elias, Amsterdam, Sociologisch Tijdschrift, 1977 ; A. Boegner, "Elias and the Francfurt School" in Theory, Culture and Society, Vol.IV, 1987 ; en particulier quand il parle de relâchement maîtrisé du contrôle des émotions (a controlled decontrolling of emotional controls).

42. Ainsi Moto Miho s'interroge-t-elle sur le fait de savoir si la politesse japonaise possède des caractères spécifiques, et pour cela elle s'intéresse à la langue japonaise. Elle remarque tout d'abord que la majeure partie des mots signifiant la politesse sont d'origine chinoise tandis que ceux qui expriment la violence ont une origine japonaise. Elle distingue aussi des mots d'origine japonaise qui concernent l'éducation concrète de l'attitude corporelle et d'autres termes plus abstraits, d'origine chinoise, qui concernent les liens avec autrui en société. On ne peut s'empêcher de songer ici à Montesquieu qui insistait sur le rôle crucial de la civilité chez les législateurs chinois, lesquels y voyaient le moyen le plus sûr de lutter contre la violence et de faire vivre le peuple en paix. C'est donc un terme d'origine chinoise "reigi" nous apprend Miho qui 
signifie politesse. L'idéogramme de rei, signifie le "rite", la "manière d'accomplir le rite" et "les normes de la vie sociale" ; celui de gi signifie "l'homme" et "l'élégance", "l'allure distinguée". L'auteur examine alors les significations de rei et de gi selon qu'ils se trouvent employés ensemble ou indépendamment. Seul, gi veut dire la "justice"... "avec rei il suggère que le visage, silhouette ou gestes élégants et policés doivent servir de modèle à tous dans la vie en société. Si rei et gi renvoient tous deux à la notion de norme et de code, rei évoque les attitudes et gestes concrets de la politesse, tandis que gi rappelle le principe qui fonde ce code : la justice". Miho, "L'esthétique et le kamikaze" dans La politesse, op. cit., p.135.

43. Ibid.

44. Ibid.

45. Ibid., p.139.

46. E. Goffman, Les rites d'interaction, op. cit., p.53 et p. 52 .

\section{RÉSUMÉS}

Norbert Elias fait du niveau de maîtrise de l'affectivité dans une société, un élément déterminant du processus de civilisation. C'est en effet dans la mise à l'écart de l'agressivité et de la violence par le contrôle social, ancré dans l'organisation étatique, qu'il voit la marque d'une société civilisée. Dans les règles de la civilité et de la politesse Elias veut donc voir la possibilité et le moyen de réaffirmer une éthique du comportement de soi, le concours de chaque individu au maintien de l'ordre dans une société. Elias n'est guère arrêté sur le fait que les règles de la civilité sont des formes qui non seulement entravent la violence mais parfois aussi la font naître et l'accompagnent. Il ne s'est pas montré très sensible à la dimension paradoxale de la " retenue ». Or, c'est un des aspects par où prolonger et approfondir la composante anthropologique et politique de la maîtrise de soi, comme l'on bien souligné les auteurs contemporains.

\section{INDEX}

Mots-clés : histoire, sociologie, sociologie historique, violence 